\title{
Evaluation of Light Activation on In-office Dental Bleaching: A Systematic Review
}

\author{
Sara Alshammery
}

\begin{abstract}
Aim: This review was undertaken to evaluate the efficacy of light activation on in-office dental bleaching in terms of color change and photosensitivity with available literature evidence.

Materials and methods: The articles were searched from MEDLINE/Pubmed and Journal of Web, which were published from 2001 to 2019.

Results: Out of the 124 references obtained, 13 articles in English language were read in full, which fulfilled the inclusion criteria.

Conclusion: Light activation of hydrogen peroxide on in-office dental bleaching does not affect effectiveness of bleach.

Clinical significance: Though claimed that in-office bleaching activated with light betters color change, the present review did not confirm this belief.

Keywords: Color change, Dental vital bleaching, Hydrogen peroxide, In-office dental bleaching, Sensitivity.

The Journal of Contemporary Dental Practice (2019): 10.5005/jp-journals-10024-2676
\end{abstract}

\section{INTRODUCTION}

Dental esthetics and smile play a significant factor in dental treatment seeking, resulting in patient demand for tooth bleaching. Early men too experimented with different chemicals to whiten their teeth. ${ }^{1}$ The first commercially marketed whitener was $10 \%$ carbamide peroxide used way back in $1989 .^{2}$ Various products and technology are described for bleaching teeth such as differing concentrations of agents, application times, and modes and the activator used with the bleaching agent. ${ }^{3}$ Dental vital bleaching is available in three categories as in office bleaching (professionally administered), over-the-counter bleaching (self-administered), and dentist-supervised take-home bleaching (professionally dispensed). Of these, the in-office involves direct dentist's supervision in order to avoid soft tissue trauma, gel ingestion, decreased therapy time, and to yield a rapid whitening result. Patients today desire to visualize the outcome of bleaching immediately, which has resulted in usage of higher concentrations of chemicals added as whiteners with different light sources. These are thought to accelerate the bleaching process. Various sources are used as light activating sources to activate bleaching process like Light emitting diodes (LEDs), Lasers, Plasma arc lamps (PAC) and halogen lamps. The principle of using a light source is that it heats the hydrogen peroxidase (HP), thereby increasing the HP's disintegration into free radicals that oxidize the complex organic molecules. ${ }^{4}$

Though light activation of vital bleaching is documented in literature, the effectiveness has been questioned by many authors. ${ }^{4-6}$ Also, tooth sensitivity is reported in several studies due to the release of excess free radicals that reach the pulp. ${ }^{7-10}$ Doubts have been raised regarding the role of these activators in increasing efficiency without causing injuries to tooth, periodontium, and oral soft tissues. Hence, this review was undertaken with the objective to evaluate the efficacy of a light source on in-office vital bleaching of tooth.

\section{Materials and Methods}

An independent researcher explored the electronic databases (MEDLINE via Pubmed) and citation databases (Scopus and Web
Restorative Department, Riyadh Elm University, Riyadh, Kingdom of Saudi Arabia

Corresponding Author: Sara Alshammery, Restorative Department, Riyadh Elm University, Riyadh, Kingdom of Saudi Arabia, Phone: +966 504186949, e-mail: sara@riyadh.edu.sa

How to cite this article: Alshammery S. Evaluation of Light Activation on In-office Dental Bleaching: A Systematic Review. J Contemp Dent Pract 2019;20(11):1355-1360.

Source of support: Nil

Conflict of interest: None

of science). Reference checks of the primary studies were made for further relevant publications. Terms used for the search were in-office bleaching, dental bleaching, vital bleaching, and light source.

Clinical studies (in vivo and in vitro) conducting in-office dental bleaching with light on adult patients, published between 2000 and January 2019, were included.

\section{Eligibility Criteria}

Clinical trials of parallel design and split mouth design, which evaluated in-office dental bleaching with or without light activation, on patients were included. Only articles in full, published in English language, were considered. Randomized trials using a combination of both home bleaching and in-office bleaching were excluded. Dissertations, theses, or abstracts in any annual conferences were not included. Articles eligible for the review were given a separate ID, comprising the first author and the year of study. Parameters of color comparison and tooth sensitivity were noted (Table 1).

\section{Risk of Bias in Selected Studies}

In order to have a quality check on selected clinical trials, two independent reviewers assessed the risk of bias using the Cochrane Collaboration tool. The assessment criteria were concealment of group allocation, blinding of outcome parameters, incomplete 


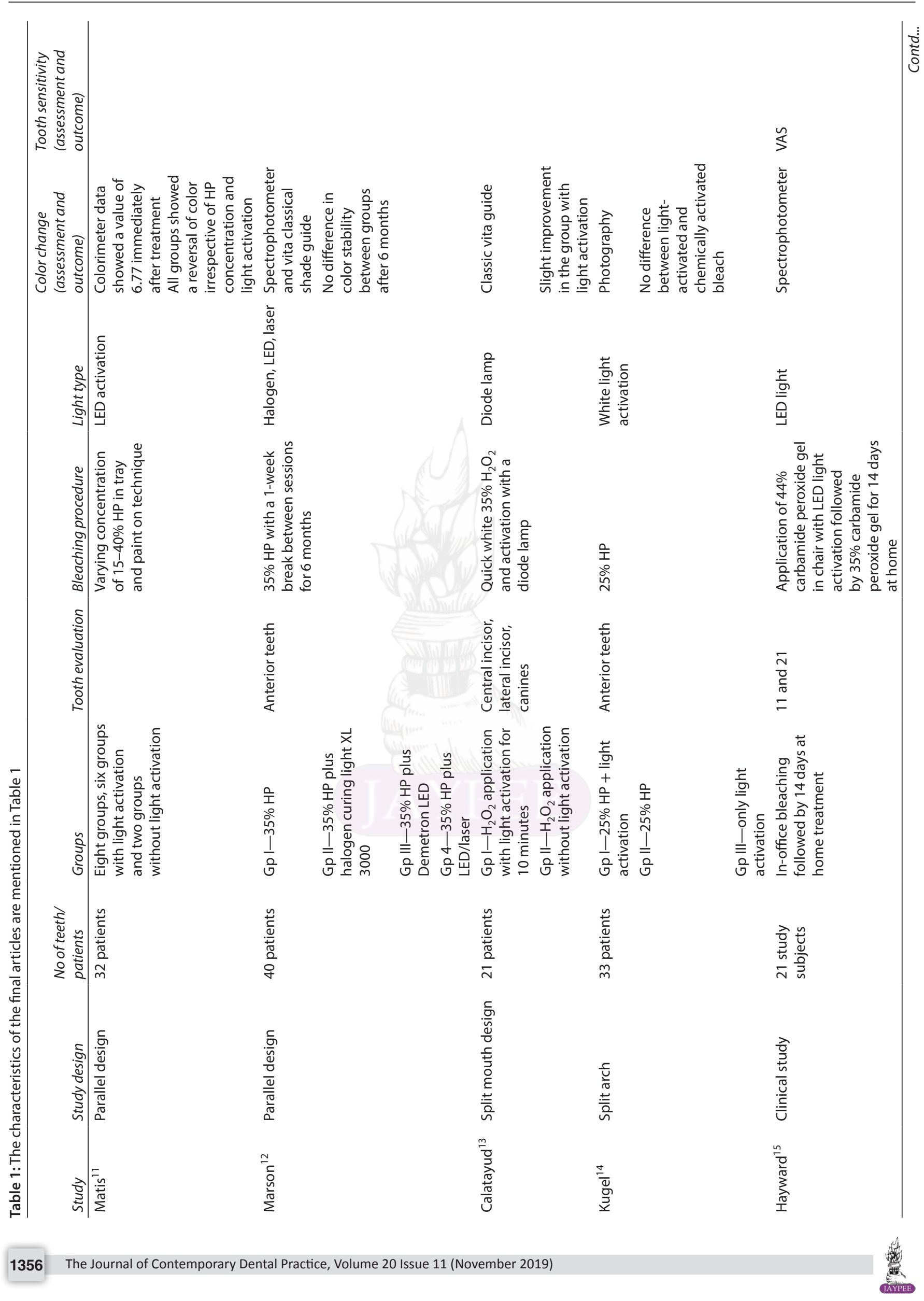




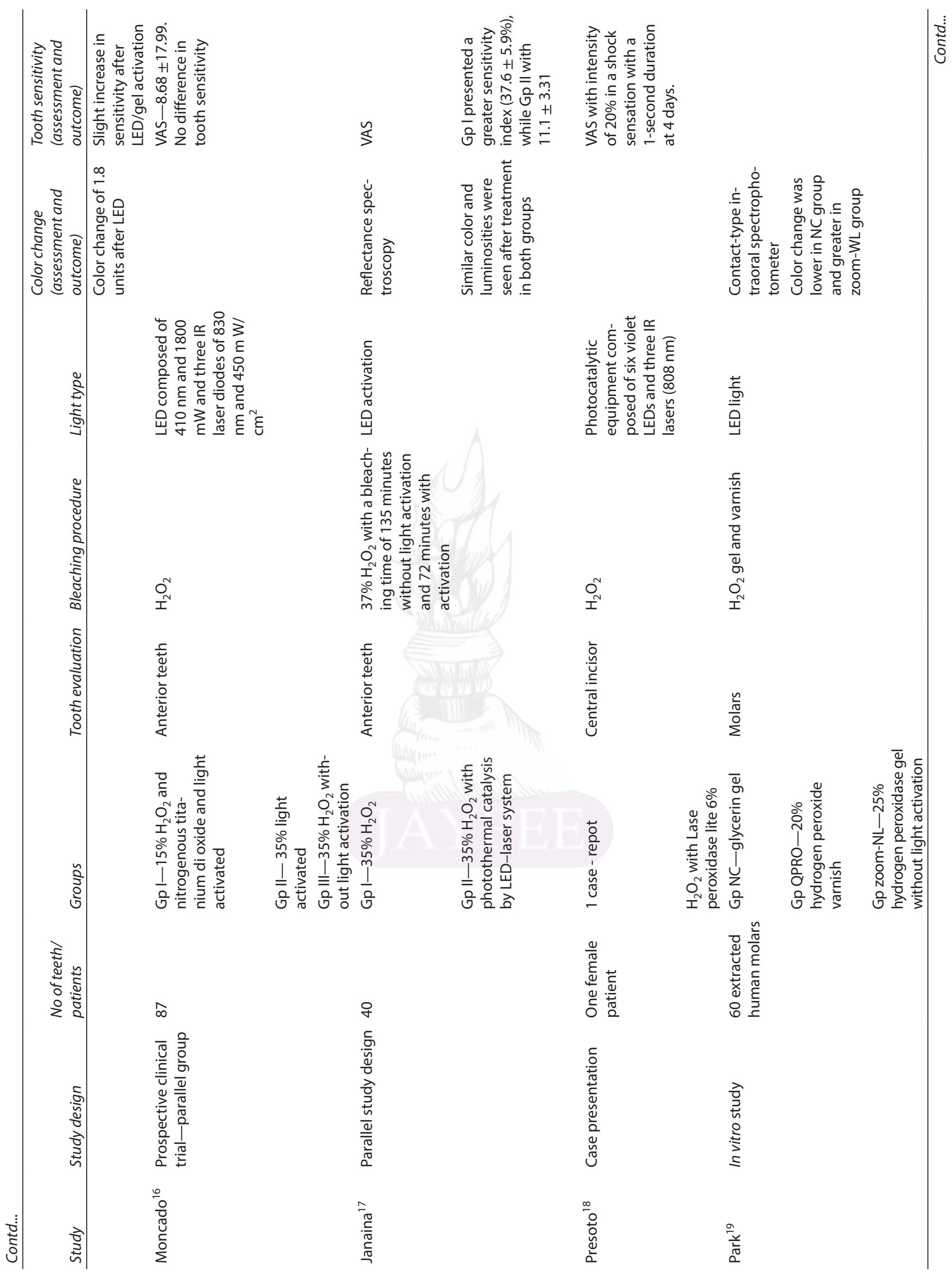




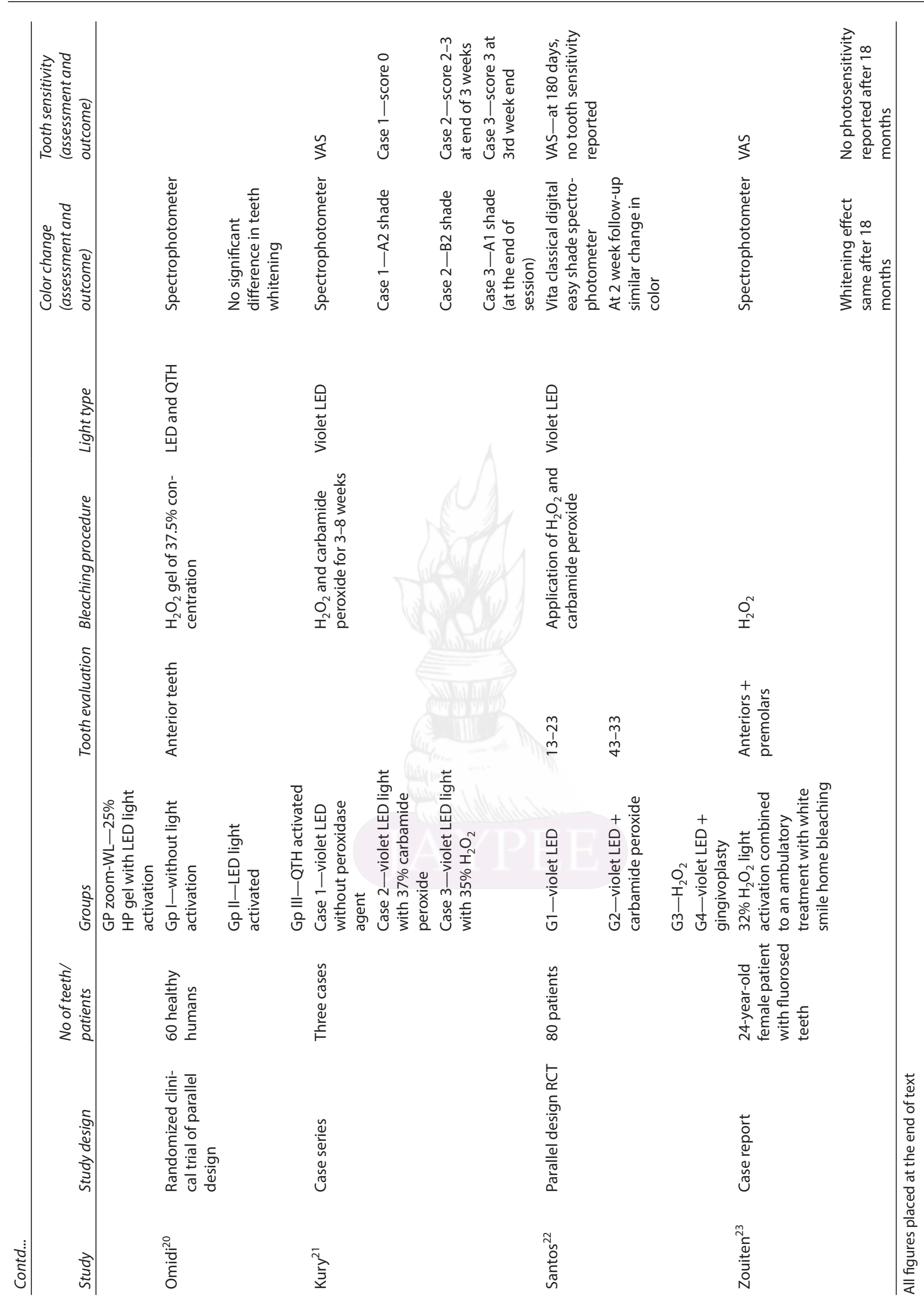


outcome data, and reporting only selective outcomes. Any disagreements between the reviewers were sorted through discussion. Each criterion was judged as having either a low, high, or unclear risk of bias. If all the outcomes had a low risk of bias, then the study was considered to be of low risk. If any outcome presented an unclear risk, then the authors were contacted to consider the study into either high risk or low risk.

\section{Results}

The search strategy was completed in January 2019, with duration of search lasting for 4 months (October 2018-January 2019). A total of 124 studies were identified in the database of which 22 were excluded as they did not meet the eligibility criteria of the review. Of the 102 articles obtained, 26 articles were excluded after the titles were read to include 76 articles. Of which, finally, 13 complete articles published in English language were included in the study after reading the abstract carefully, which met the inclusion criteria of the reviewer.

Nine articles were randomized clinical trial, with two following a split mouth technique and the rest were of concurrent design parallel study, three case reports, and one was an in vitro study. The different methods of in-office activation were LED activation in seven studies, a combination of halogen LED and laser in one study, use of diode lamp in one, a combination of LED and infrared (IR) laser diode in one, violet LED activation in two, and a combination of violet LED and IR laser activation in one study (Flowchart 1).

Of the 13 articles that were reviewed, color change by light activation was slightly better in only one study (Calatayud et al.). In all the other studies, there was no significant difference in the color change between any in-office bleaching activation modality. The study results did not present any association of light activation with in-office bleaching to sensitivity.

\section{Discussion}

The current review aimed to evaluate the efficacy of lightactivated in-office dental bleaching in terms of color change

Flowchart 1: Literature search flowchart for systematic review on evaluation of light activation on in-office bleaching of tooth

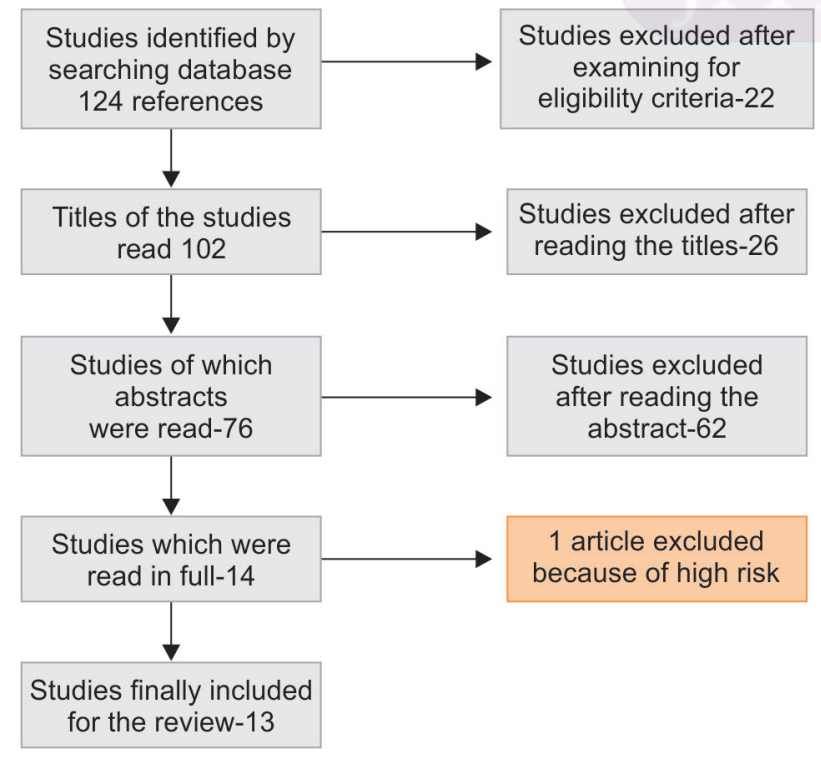

and tooth sensitivity, based on articles published between 2001 and 2019. Consensus was reached between tooth sensitivity and light activation that the latter did not result in tooth sensitivity. Out of the 76 abstracts read, 63 were excluded making the final selected articles to a total of 13 . Of the 13 articles reviewed, 9 were randomized clinical trial of which 7 had parallel design and 2 had split mouth technique, 1 was in vitro study, and 3 case reports.

Tooth sensitivity arises when hydrogen peroxide molecules pass through the enamel and dentin into the pulp chamber resulting in pulpal inflammation. This affects the sensory nerves, which in turn perpetuate an increased response to stimuli. Various factors that affect tooth sensitivity are dental cracks, dentin exposure, or dimensions of the pulp chamber. Tooth sensitivity in all studies was measured by a visual analog scale (VAS), ranging from 0 to 100 , where 0 marks no pain and 100 presents extreme pain.

Three studies in the review used violet LED for light activation and presented no photosensitivity. ${ }^{22,24,25}$ Violet LED is used as a light source to promote bleaching instead of chemical agents. Visible violet light in the wavelength of $405 \mathrm{~nm}$ is used in the absence of any peroxide agent. Violet light emits photons that propagate at smaller wavelength and higher frequency, thus exhibits less penetration into the dental tissue and hence presents decreased sensitivity. This property proves to be beneficial as it promotes breakage of the large pigment chains at low heat. ${ }^{24,25}$ The lower penetration of violet LED leads to lesser molecular alteration and lesser depth operation, thus preserving the pulp.

Three studies ${ }^{5,17,30}$ used the bleaching protocol in combination with a hybrid light (LED and laser) source. Studies have shown that the time taken for bleaching with hybrid light is lesser than LED alone. This shows that shorter clinical time is required for bleaching with a hybrid light source. Frietas et al. ${ }^{5}$ reported in their study that the group with hybrid light source activation showed considerable reduction in pulp inflammation. Although the low-power laser demonstrates anti-inflammatory and biomodulation potentials, still no scientific evidence of its effect on reducing post-bleaching sensitivity is proven.

The study results did not present any association of light activation with in-office bleaching to sensitivity. This is in concordance with the results of a systematic review by Ray ${ }^{26}$ done on 11 studies that produced weak evidence to the use of light activation with in-office vital bleaching. Regardless of the HP concentration used, light activation used increased tooth sensitivity. Hypothetically, both heat and light sources accentuate the disintegration of hydrogen peroxide to form oxygen and perhydroxy free radicals, which are thought to increase bleaching efficiency. ${ }^{27,28}$

In-office bleaching has several advantages like it is a quick procedure and less riskier as performed by procedure. It also presents certain disadvantages like it is expensive and results are unpredictable as they are dependent on several factors. The final result of bleaching is dependent on patient's age, original tooth color shade, concentration of bleaching agent, and the time factor.

A study done by Hahn et al. ${ }^{29}$ reported that light activation of $\mathrm{HP}$ for bleaching does not prove beneficial as the color is not stable after 3 months, and that the increase in temperature can affect pulp. Torres et al. ${ }^{30}$ also opine that though photoactivation results in fast bleaching, color regression was observed in less than a year.

The review has two elements of error owing to the subjective analysis of color assessment (shade guide) as the same evaluator can interpret different shades at different times and the objective 
analysis of the spectrophotometer, which can give varied results because of mirror changes.

Further studies are recommended to reach a definite consensus on the efficacy of light activation on in-office dental bleaching by using standardized protocols for research like standard concentration of bleaching material, standard application time, assessment time, and duration of the bleaching effect.

\section{Conclusion}

The present review provides no positive association of light activation of in-office dental bleaching with either increasing efficacy or the risk of tooth sensitivity. The result needs to be interpreted with caution considering the variations in procedure, process, and techniques (hydrogen peroxide concentration, bleaching sessions, light activation sources, and products used).

\section{References}

1. Khin PW, Barnes DM, Romberg E, et al. A clinical evaluation of $10 \%$ vs $15 \%$ carbamide peroxide tooth whitening agents. J Am Dent Assoc 2000;131:1478-1484. DOI: 10.14219/jada.archive.2000.0061.

2. Sulieman M, Addy M, Mac Donald E, et al. The effect of hydrogen peroxide concentration on the outcome of tooth whitening: an in vitro study. J Dent 2004;32:295-299. DOI: 10.1016/j.jdent.2004. 01.003.

3. Ben Cheikh Ahmed H, Gnaba I, Smaoui M, et al. In office tooth bleaching treatment using light - activated hydrogen peroxide agent combined with ambulatory treatment: A case report. Sci Arch Dent Sci 2019;2(2):22-27.

4. Maran BM, Burey A, De Paris Matos T, et al. In office dental bleaching with light vs. without light. A systematic review and meta-analysis. J Dent 2018;70:1-13. DOI: 10.1016/j.jdent.2017.11.007.

5. De Freitas PM, Menezes AN, Da Mota AC, et al. Does the hybrid light source (Led/Laser) influence temperature variation on the enamel surface during $35 \%$ hydrogen peroxide bleaching? a randomized clinical trial. Quintessence Int 2016;47:61-73.

6. Sulieman M, Macdonald E, Rees JS, et al. Comparison of three in-office bleaching systems based on $35 \%$ hydrogen peroxide with different light activators. Am J Dent 2005;18:194-197.

7. Ontiveros JC, Paravina RD. Color change of vital teeth exposed to bleaching performed with and without supplementary light. J Dent 2009;37:840-847. DOI: 10.1016/j.jdent.2009.06.015.

8. Kossatz S, Dalanhol AP, Cunha T, et al. Effect of light activation on tooth sensitivity after in-office bleaching. Oper Dent 2011;36:251-257. DOI: 10.2341/10-289-C.

9. Trindade FZ, Ribeiro AP, Sacono NT, et al. Trans-enamel and transdentinal cytotoxic effects of a $35 \% \mathrm{H}_{2} \mathrm{O}_{2}$ bleaching gel on cultured odontoblast cell lines after consecutive applications. Int Endod J 2009;42:516-524. DOI: 10.1111/j.1365-2591.2009.01544.x.

10. Kugel G, Ferreira S, Sharma S, et al. Clinical trial assessing light enhancement of in-office tooth whitening. J Esthet Restor Dent 2009;21:336-347. DOI: 10.1111/j.1708-8240.2009.00287.x.

11. Matis BA, Cochran MA, Franco $M$, et al. Eight in-office tooth whitening systems evaluated in vivo: A pilot study. Oper Dent 2007;32(4): 322-327. DOI: 10.2341/06-135.
12. Marson FC, Sensi LG, Vieira LC, et al. Clinical evaluation of in-office dental bleaching treatments with and without the use of light activation sources. Oper Dent 2008;33:15-22. DOI: 10.2341/07-57.

13. Calatayud JO, Calatayud CO, Zaccagnini MS, et al. Clinical efficacy of a bleaching system based on hydrogen peroxide with or without light activation. Eur J Esthetic Dent 2010;5:216-224.

14. Kugel G, Papathanasiou A, Williams AJ, et al. Clinical evaluation of chemical and light-activated tooth whitening systems,. Compend Continuing Edu Dent 2006;27:54-62.

15. Hayward R, Osman Y, Grobler SR. A clinical study of the effectiveness of a light emitting diode system on tooth bleaching. Open Dent J 2012;6:143-147. DOI: 10.2174/1874210601206010143.

16. Moncado G, Sepulveda D, Elphick K. Effects of light activation, agent concentration and tooth thickness on dental sensitivity after bleaching. Oper Dent 2013;38(5):467-476. DOI: 10.2341/12-335-C.

17. Bortolatto JF, Pretel $H$, Neto CS, et al. Effects of LED laser hybrid light on bleaching effectiveness and tooth sensitivity: a randomized clinical study. Laser Phys Leet 2013;10:1-7.

18. Presoto CD, Bortolatto JF, de Carvalho PPF, et al. New Parameter for in - office dental bleaching. Case Rep Dent 2016; 1-4.

19. Park SY, Kwon SR, Qian F, et al. The effect of delivery system and light activation on tooth whitening efficacy and hydrogen peroxide penetration. J Esthet Restor Dent 2016;28(5):313-320. DOI: 10.1111/ jerd.12238.

20. Omidi BR, Nouri M, Farahmandpour N. Evaluation of two types of bleaching gel and light source on whitening of teeth. Biosci Biotech Res Comm 2017;10(3):564-570. DOI: 10.21786/bbrc/10.3/34.

21. Kury M, Resende BA, da Silva DP, et al. Clinical application of violet LED in - office bleaching with or without traditional systems: Case series. Oral Health and Dent Stud 2018;2(1):1.

22. Santos AECG, Bussadori SK, Pinto MM. Evaluation of in-office tooth whitening treatment with violet LED: protocol for a randomised controlled clinical trial. BMJ Open 2018;8(9):e021414. DOI: 10.1136/ bmjopen-2017-021414.

23. Zouiten S, Ben Cheikh Ahmed H, Gnaba I, et al. In office tooth bleaching treatment using light activated hydrogen peroxide agent combined with ambulatory treatment: A case report. Sci Arch Dent Sci 2019;2(2):22-27.

24. Zanin F. Recent advances in dental bleaching with laser and LEDs. Photomed Laser Surg 2016;34:135-136. DOI: 10.1089/pho.2016.4111.

25. Panhoca VH, Oliveira BP, Rastelli ANS, et al. Dental bleaching using violet light alone: clinical case report. Dentistry 2017; 7-11.

26. Ray DS. Using light to enhance in-office vital bleaching may increase both efficacy and tooth sensitivity. J Am Dent Assoc 2014;145(11): $1159-1160$.

27. Joiner $A$. The bleaching of teeth: a review of the literature. J Dent 2006;34:412-419. DOI: 10.1016/j.jdent.2006.02.002.

28. Kashima-Tanaka M, Ysujimoto Y, Kawamoto K, et al. Generation of free radicals and/or active oxygen by light or laser irradiation of hydrogen peroxide or sodium hypochlorite. J Endod 2003;29:141-143. DOI: 10.1097/00004770-200302000-00013.

29. Hahn P, Schondelmaier N, Wolkewitz M, et al. Efficacy of tooth bleaching with and without light activation and its effect on the pulp temperature: an in vitro study. Odontology 2012;101(1):67-74.

30. Torres CR, Barcellos DC, Batista GR, et al. Assessment of the effectiveness of light emitting diode and diode laser hybrid light sources to intensity dental bleaching treatment. Acta Odontol Acand 2011;69:176-181. DOI: 10.3109/00016357.2010.549503. 